\title{
Filigrane
}

Écoutes psychanalytiques

\section{Toxicomanie et grossesse : enjeux des remaniements psychiques de la grossesse sur la problématique addictive}

\section{Anaëlle Bazire et Nadine Proia-Lelouey}

Volume 25, numéro 1, printemps 2016

Actes de la journée clinique de la SPQ (Catherine Chabert)

URI : https://id.erudit.org/iderudit/1037380ar

DOI : https://doi.org/10.7202/1037380ar

Aller au sommaire du numéro

Éditeur(s)

Revue Santé mentale au Québec

ISSN

1192-1412 (imprimé)

1911-4656 (numérique)

Découvrir la revue

Citer cet article

Bazire, A. \& Proia-Lelouey, N. (2016). Toxicomanie et grossesse : enjeux des remaniements psychiques de la grossesse sur la problématique addictive.

Filigrane, 25(1), 147-165. https://doi.org/10.7202/1037380ar 


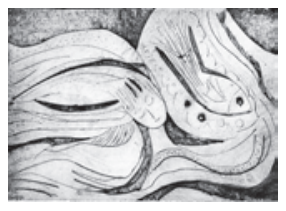

\title{
Toxicomanie et grossesse: enjeux des remaniements psychiques de la grossesse sur la problématique addictive
}

\author{
Anaëlle Bazire \\ et Nadine Proia-Lelouey
}

\begin{abstract}
Les auteures évoquent les résultats d'une recherche académique sur les conséquences des remaniements psychiques induits par la grossesse sur la problématique addictive. À partir de données issues d'entretiens et de tests de Rorschach auprès de femmes toxicomanes enceintes, elles mettent en évidence en quoi les bouleversements psychodynamiques induits par la grossesse permettent une relance des impasses psychiques en jeu dans l'addiction. Elles confirment les données de la littérature sur le potentiel transformatif de la grossesse psychique sur la problématique addictive, mais soulignent que ce potentiel n'est pas équivalent pour toutes les femmes, qu'il n'est pas linéaire et reste très fragile.
\end{abstract}

a maternité des femmes dépendantes aux substances psychoactives est - une question de santé publique et fait progressivement l'objet depuis quelques années de la mise en place de structures spécifiques ou de réseaux pluridisciplinaires ${ }^{1}$. On estimait, il y a dix ans, le nombre de toxicomanes en France dans une fourchette de 150000 et 300000 personnes avec 25 à $30 \%$ de femmes, dont une large majorité en âge de procréer (Lejeune, 2007). De fait, la possibilité d'une grossesse chez une femme usagère de drogue est à l'évidence fréquente. Plus précisément, selon Franchitto, Peyrefort et Tellier (2010), 500 à 2500 grossesses sont menées à terme chaque année en France par des femmes toxicomanes. Toubiana (2011) évoque quant à lui, 2400 à 5000 naissances sous toxicomanie par an. Ces femmes sont fréquemment polyconsommatrices avec une prévalence de dépendance aux opiacés (héroïne ou traitements de substitution), associée en général à des consommations de tabac, cannabis, cocaïne ou médicaments psychotropes (Lejeune, 2007).

Des études sur les femmes enceintes toxicomanes existent mais portent essentiellement sur des questions médicales ou psychosociales (Lejeune, 
2007; Molénat, 2000; Simmat-Durand, 2002). Très peu de travaux abordent la question d'un point de vue psychodynamique et quelques-uns seulement sur l'articulation entre les remaniements psychiques propres à la grossesse et la problématique addictive. Pourtant, il semble important de développer des programmes de recherche sur cette question afin d'améliorer la prise en charge de ces femmes grâce à une meilleure prise en compte des dynamiques psychiques en jeu.

Dans une première étude, nous avons exploré les éventuelles interférences entre les bouleversements psychiques induits par la grossesse et l'homéostasie psychique créée par le lien au produit.

La grossesse constitue un moment évolutif fondamental pour la femme, lié à la réactualisation — et à l'éventuelle réélaboration — de conflits infantiles (Bibring, 1959). Elle entraine d'importants remaniements psychiques (Bydlowski, 1997; Molénat, 2000) qui s’avèrent étroitement liés aux problématiques présentes dans la toxicomanie. Soulignons tout particulièrement le conflit narcissico-objectal, les troubles du moi corporel et l'enjeu des limites (Touzanne, 2009), ou encore, la question du processus de séparationindividuation (Pines, 1972). Ces éléments, indispensables à la construction du devenir sujet de chaque individu, restent en souffrance chez les sujets toxicomanes alors même qu'ils sont réactualisés par la maternité. De ce fait, une grossesse chez une femme addicte constitue une situation de particulière vulnérabilité. Cependant, elle peut aussi être un moment potentiellement transformatif. La grossesse pourrait alors s'envisager comme une période durant laquelle une sortie de la toxicomanie serait possible si elle s'accompagne d'une prise en charge adaptée.

\section{Méthodologie de l'étude}

Par le biais de maternités ou de centres spécialisés dans la prise en charge des addictions, nous avons été mises en contact avec des femmes enceintes toxicomanes. Dix ont répondu favorablement à l'idée de participer à notre étude et six d'entre-elles, consommatrices d'opiacés ou sous traitement de substitution aux opiacés (TSO), ont donné suite. Nous avons rencontré ces futures mères à leur septième mois de grossesse ${ }^{2}$. Nous leur avons proposé un entretien semi-directif ainsi que la passation d'un Rorschach. Notre analyse du test projectif (à l'aide du système de cotation de l'École de Paris) était centrée sur la relation précoce de la femme à sa propre mère et sur l'accès à l'identification sexuée. Nous avons dans un premier temps — selon la démarche traditionnelle de l'étude de cas — rassemblé les données issues 
des entretiens et des tests projectifs afin d'établir une analyse clinique pour chaque sujet avant de rassembler les données cliniques transversales congruentes et divergentes que nous avons confrontées à la littérature.

En annexe, le lecteur trouvera une présentation générale des sujets.

\section{Grossesse et modifications corporelles}

Franchitto et al. (2010) avancent l'idée que les femmes toxicomanes attribuent les changements corporels et les sensations dues à la grossesse à la drogue et méconnaissent leur état gravidique. Nous n'avons retrouvé cette configuration que pour une seule de nos six sujets, Charlotte ${ }^{3}$. Les cinq autres femmes ont eu connaissance de leur grossesse durant le premier mois suite aux changements corporels (seins lourds), aux manifestations physiologiques (p. ex., des vomissements) ou à l'aménorrhée. Ce qui laisse penser qu'elles manifestent un investissement de leur corps et de leur image corporelle suffisant. Quatre des six grossesses (pour Karine, Natasha, Charlotte et Véronique) étaient d'ailleurs désirées bien que non-programmées au moment où elles ont eu lieu (Lacrouts-Négrier, Roux, Taboada, et Schmelck, 2001).

C'est voulu dans le sens où je veux des enfants mais pas maintenant en fait. (Karine)

Cependant, pour Cécile, le désir d'enfant semblait plus lié à un collage à la norme sociale, la grossesse étant représentée comme un "passage obligé» lié à son avancée en âge. Les deux dernières grossesses n'avaient jamais fait l'objet d'un projet d'enfant. Zoé a d'ailleurs souhaité avorter mais le père de l'enfant désirait qu'elle poursuive cette grossesse. Au fil de l'entretien, il apparaîtra qu'elle a finalement gardé l'enfant davantage pour s'assurer du lien avec son conjoint que dans un désir réel d'enfant.

Les symptômes physiques tels que les vomissements mais aussi les modifications corporelles vécues comme subies, ou encore le sentiment d'être limitée par son corps dans ses activités peuvent renvoyer, comme pour les femmes enceintes non toxicomanes, à l'ambivalence vis-à-vis de la grossesse et de la présence de l'autre en soi (Lacrouts-Négrier et al., 2001).

[La grossesse] m'handicape un peu [...] Au début il m’a fallu un petit temps pour que je m'adapte en fait. Que je me dise «mon corps va changer». (Véronique) 
Mais ces manifestations renvoient également à la question de la passivité, inhérente à la féminité et tout particulièrement à la grossesse, qui peut être vécue dans le registre de la passivation. Ce concept de Green (1990) rend compte des vécus primaires de la passivité dans les soins maternels. Ceux-ci peuvent permettre au sujet d'accéder à une "passivité bien tempérée» (Proia-Lelouey et Schvan, 2011) et ouvrir à la symbolisation ou rester en souffrance et entraver tout le travail de représentance (Green, 1999). Ainsi, si la passivation s'avère un concept particulièrement opérant pour rendre compte de la problématique addictive telle qu'elle est présentée par la littérature ${ }^{4}$, les modifications liées à la grossesse viennent réactiver ces vécus de passivation et donc, redoubler la problématique toxicomane.

De fait, nous avons pu repérer chez Cécile et Zoé, une opposition massive face aux changements induits par la grossesse.

Y’a pas de changements. (Cécile)

[...] enfin j'avais que les symptômes mais sinon euh j'grossissais pas, y'avait rien du tout qui changeait donc euh j'faisais comme si... comme si y'avait rien. (Zoé)

Il est probable que ces remaniements et tout ce qu'ils impliquent (reviviscence des conflits infantiles, réélaboration du processus de séparationindividuation, ambivalence, plus grande perméabilité aux représentations inconscientes du fait de la transparence psychique) soient vécus sur le registre de la passivation et donc trop effractants. (Cohen-Salmon Missonnier et Marty, 2011). Ils traduiraient une réactivation pulsionnelle induite par la centration vers l'intériorité due à la grossesse menaçant la femme d'un sentiment de perte d'identité et de la continuité d'existence (Cohen-Salmon et al., 2011). Le processus de temporalité induit par la grossesse se trouve alors compromis avec ce qu'il implique de travail sur la perte mais aussi de reconnaissance de l'enfant comme individualité propre. La lutte contre les changements somatopsychiques rend compte également d'une angoisse de perdre le contact avec la réalité étroitement liée au vécu de passivation.

Le refus massif des changements inhérents à la grossesse implique au final, chez ces deux femmes, un refus de la dynamique mutative proposée par la grossesse (Rosenblum, 2009). L'enfant n'aura pas entrainé un renflouage narcissique suffisant pour permettre un arrêt de la drogue qu'elles ont continuée à consommer, en plus du traitement, pendant la grossesse 
(Nezelof et Dodane, 2010). Ces consommations durant la grossesse peuvent d'ailleurs être vues comme un moyen de mettre à distance et gérer les ressentis négatifs et effractants liés à la présence de l'autre en soi (Cohen-Salmon et al., 2011). Il semble donc que la grossesse comme processus transformatif permettant une atténuation de la dépendance et une mobilisation de l'investissement libidinal et objectal soit dans l'impasse chez Cécile et Zoé. Pour nos quatre autres sujets, la difficulté à accepter les changements inhérents à la grossesse est beaucoup moins massive et laisse penser que les enjeux de passivité sont, pour ces dernières, moins traumatiques. Nous pensons par ailleurs que la question du désir d'enfant se pose différemment dans les deux groupes de femmes.

Pour Bydlowski (1997), il existerait chez toute femme, même si elle refuse la maternité, un désir inconscient d'enfant lié au désir de l'enfant imaginaire supposé tout réparer et tout combler (deuil, solitude, destin, sentiment de perte ou de vide). Cependant, ce désir, chez les femmes toxicomanes, pourrait se voir «court-circuité» par la drogue. La survenue d'une grossesse serait alors le signe d'une rupture de cette homéostasie. Ainsi, Rosenblum (2009) considère que l'objet-drogue serait insuffisant pour soutenir l'économie psychique des jeunes femmes, et Ebert (1988) estime que la grossesse serait le signe d'une tentative de résolution des conflits inconscients à l'origine de la toxicomanie. On retrouve cette idée chez Taboada (1998) pour qui le désir d'enfant n'apparaitrait que lorsque le colmatage toxicomaniaque n'y suffit plus, avec un recours à la maternité comme tentative de guérison. Esquivel (1994), citant Sainte-Fare-Garnot (1986), est plus précise et considère que lorsque le désir d'une maternité est présent consciemment, l'enfant apparaît comme l'aboutissement d'une maturation psychique de bon augure.

Pour avancer sur cette question, il paraît important de faire le distinguo entre recours à la maternité et désir de maternité. Pour nos six sujets, la grossesse est «accidentelle». Recours à l'acte ou acte manqué? Question fondamentale au regard de la problématique addictive, car le premier processus montre que le sujet est toujours dans l'économique et le besoin, alors que le second signale la mise en acte d'un souhait certes refoulé mais relevant du registre du désir. Quatre de nos sujets ont fait état d'un désir d'enfant, celleslà même qui ont fait preuve d'une meilleure tolérance aux changements corporels. On peut donc raisonnablement penser qu'elles sont davantage dans la mise en acte d'un désir inconscient et que Cécile et Zoé, qui n'ont jamais évoqué un quelconque désir de maternité, seraient davantage dans le recours à l'acte. 
Mais les choses ne sont pas si simples car c'est Charlotte qui a confondu sensations dues à la drogue et à la grossesse, même si elle les a bien tolérées une fois connu son état gravidique. Par ailleurs, Natascha est la seule à ne pas s'être fait suivre correctement durant sa grossesse et à avoir mis en péril son enfant en accouchant dans une maternité autre que celle prévue et non pourvue d'un service de néonatologie. Notons aussi que Zoé a déjà une fillette et que sa toxicomanie a commencé un an environ après la naissance de celle-ci.

On ne peut donc souscrire à l'idée quelque peu irénique qu'un désir d'enfant et une maternité viendraient résoudre la question de la toxicomanie. Il s'agit plus modestement d'anticiper, selon les configurations psychiques des futures mères, la façon dont vont s'articuler les liens à l'objet-drogue et les liens à l'enfant. En d'autres termes, peut-on penser que la meilleure tolérance aux modifications corporelles chez quatre de nos sujets signe une meilleure tolérance à la réceptivité et la construction d'une espace psychique de contenance avec un bébé différencié (Missonnier, 2004)?

\section{Représentations du bébé}

La perception des mouvements foetaux au $4^{\mathrm{e}}$ ou $5^{\mathrm{e}}$ mois de grossesse - et les échographies - ont largement contribué chez ces quatre sujets à l'élaboration de représentations d'un bébé reconnu comme un être d'altérité.

J'ai commencé à sentir un petit être qui bouge [...] tant qu'on n'a pas vu, on n'y croit pas, $[\ldots]$ on le sait mais on a rien vu, [...] [Je] voyais un petit être se former, bah oui, c'est bel et bien mon bébé et dans 9 mois il est là quoi. (Véronique)

Les mouvements foetaux signent la fin de la fusion (Bayle, 2005). Ils ont permis chez elles, comme chez les femmes enceintes non toxicomanes, une figuration du bébé (Cohen-Salmon, 2011). La reconnaissance des mouvements fotaux marque également la mise en jeu des sensations corporelles dans le travail de symbolisation puisqu'elle participe à la création de représentations du bébé (Rosenblum, 2009).

La petite elle a la bougeotte. Elle a une force énorme. Et je sais qu'elle va être super musclée parce qu'elle nous a fait beaucoup de hoquets en fait. (Charlotte) 
En revanche et contrairement aux observations de Soulé (1982) concernant les femmes non toxicomanes, les mouvements fotaux ne permettent pas la disparition de l'enfant imaginaire au profit de l'enfant «réel» pour ces futures mères. Ils semblent, au contraire, être la base de représentations d'un «bébé narcissique». On retrouve en effet chez nos sujets un bébé très idéalisé, représentant tour à tour une possibilité de repartir de zéro, une tentative de réparation narcissique, un protecteur ou un soutien (Nezelof et Dodane, 2010; Simmat-Durand, 2002).

Il donne un coup euh. Donc je sais pas si c'est inconsciemment pour me rassurer ou si c'est moi qui m'dis «tiens il donne un coup, il te rassure quoi» (Véronique)

Le risque est la non-élaboration de la différenciation du bébé qui ne sera pas reconnu comme un être d'altérité avec ses besoins et désirs propres, mais investi narcissiquement par la mère. Ce mouvement est particulièrement à l'œuvre chez Karine.

Dès que j'me mets à pleurer, ça r'bouge. J'dis tiens elle m'lance une perche pour que j'l'attrape. (Karine)

Chez elle, on constate un arrêt total de toutes les consommations pendant la grossesse (TSO, cannabis et tabac) associé à une crainte de dépendance à l'enfant à naître. Celui-ci, qui reste indifférencié, est représenté uniquement comme protecteur contre une éventuelle reprise des consommations. Il est, à ce titre un véritable «pare-drogue» (Franchitto et al., 2010) voire un objet-drogue de remplacement (Groupe Pompidou, 2001).

Néanmoins, son bébé fait tout de même l'objet, comme celui des autres futures mères rencontrées sauf Cécile, de représentations concernant son caractère ou son physique. Ces cinq sujets parlent à leur futur enfant, le nomment et ont déjà choisi le prénom. Ces relations avec l'enfant à venir marquent un investissement de celui-ci sur un mode différencié et montrent que chez elles aussi les représentations issues des mouvements fotaux permettent d'établir une relation anténatale à l'enfant (Ammaniti, Candelori et Pola, 1999). Il semble donc que la présence, nécessaire à l'établissement des relations postnatales, d'un espace psychique maternel d'interaction et de contenance avec un bébé investi et reconnu comme un être d'altérité soit bien présente. Propos qu'il faut néanmoins nuancer car si la présence 
du double mouvement conflictuel — maintien d'un objet narcissique versus autrui reconnu dans son altérité — signale bien la réactualisation des processus de séparation-individuation, du conflit narcissico-objectal et des modalités de relation d'objet, rien ne dit que cette tentative de les dépasser et de les résoudre (Franchitto et al., 2010) aboutisse.

Cette conflictualité n'est pas atteinte par Cécile dont l'enfant ne fait l'objet d'aucune représentation. Lorsque nous lui demandons comment elle imagine son enfant, elle nous dit «ne pas y avoir pensé». Finalement elle verbalisera qu'elle l'imagine «comme nous [elle et son conjoint] quoi mais autrement... non.». Elle qualifie par ailleurs les mouvements foetaux de quelque chose de «bizarre» et plutôt négatif. Ces mouvements ont néanmoins, semble-t-il, permis de prendre conscience qu'elle avait un enfant en elle. Reste qu'elle ne l'imagine que comme un double de soi et de son conjoint dans un collage narcissique qui souligne l'absence d'élaboration du processus de séparation-individuation et de changements concomitants des modalités de relations objectales. Constat qui fait redouter des conséquences délétères sur l'établissement des relations post-natales et le développement psychique du bébé (Missonnier, 2004).

\section{Angoisses de mort sur le bébé}

Les mouvements foetaux, comme chez les autres femmes, rassurent nos sujets sur la santé de leur bébé.

Dès qu'elle bouge, j'me dis elle est là, ça va. [...] Dès qu'j'le sens plus bouger, ben euh, j'ai peur. [...] Ben j'suis contente parce que moi qui croie toujours qu'elle est morte euh. (Natasha)

Nous constatons malgré tout des angoisses massives de mort et de malformations du fotus. Angoisses que l'on peut retrouver chez toutes les femmes enceintes ${ }^{5}$ mais qui sont majorées chez nos femmes toxicomanes (Molénat, 2000).

Ils sont au courant que j'prenais de l'héroïne avant mais pas du traitement. Et heuuuuu. Et bah par rapport au bébé euhhh. J'ai eu peur surtout au handicap. J'ai eu super peur de ça. J'ai encore peur. J'ai peur de qu'il, qu'il meurt à la naissance. [...] J'ai juste peur qu'y'ait un problème de handicap ou de de mort à la naissance quoi. (Natasha) 
Ces angoisses signalent, pour toutes les femmes, les mouvements d'ambivalence et notamment les vœux de mort à l'égard des êtres chers. Cette ambivalence se manifeste sous la forme d'un conflit entre le souhait d'avoir un enfant sain et vivant et le désir insensé de le mettre à mal afin de faire disparaître l'enfant préœedipien et/ou œdipien qu'il incarne (Bydlowski, 1997). Mais au-delà de ces mouvements d'ambivalence, ces angoisses de mort et de malformation condensent, chez nos sujets, d'autres significations. Tout d'abord elles se représentent comme toxiques pour leur enfant.

La pauvre, s'ils m'font accoucher, c'est déjà qu'elle est pas bien dans mon ventre. (Karine)

Elles font aussi état d'une angoisse massive de séparation qui apparaît chez Karine par exemple, sous forme de contre-investissement.

J'dis j'veux pas être esclave d'un enfant. [...] J'dis, je veux [à la] limite on m'enlève ma fille de force au bout de même trois, quatre mois. C'est pas méchamment, c'est pour l'habituer à c'qu'elle aille partout. (Karine)

Enfin, l'angoisse de mort ou de malformation comme on l'a illustrée pour Natasha ci-dessus peut traduire une formation réactionnelle de vœux de mort envers le fotus vécu comme une menace pour leur intégrité et leur identité.

Ces angoisses sont par ailleurs toujours associées à une culpabilité massive (Taylor, 1993, cité dans Guyon, De Konink, Morissette, et Chayer, 1998) qui apparait en creux dans le discours de ces femmes. On la repère tout particulièrement dans divers mécanismes de défense comme les projections sur autrui ou dans la réalité.

Mais elle [son amie] a picolé tous les weekends quand même. Elle a pris de la coke tous les weekends, quand y'avait pas de coke c'était du MDMA, c'est carrément pire. (Karine)

Ou encore, on remarque le déni plus ou moins massif de leurs consommations et de leurs conséquences sur le foetus.

J'ai fait une grosse soirée, tout ça euh... Bon y'a pas eu de conséquences hein, le bébé était encore là. [...] Non quand je consomme, parce que déjà 
je prends pas de cocaïne parce que je sais que c'est très... c'est encore plus dangereux que la came. [...] j'me bloque à si je rechute, j'rechute que avec ça [héroïne] et encore euh... voilà j'en ai pris... j'en ai pas pris non plus pendant toute une soirée encore. C'était en journée et euh... j'étais pas vraiment euh... J'étais pas plus dans un bon état qu'autre chose donc euh... (Zoé)

La culpabilité se repère également dans les inquiétudes quant au risque de syndrome de sevrage du nouveau-né à la naissance ${ }^{6}$.

C'est clair que je me sens coupable à fond quoi, qu'elle soit obligée d'avoir un truc à cause de moi. Un traitement. (Natasha)

Selon Rosenblum (2009) la culpabilité liée aux conséquences des consommations chez les femmes enceintes toxicomanes, témoignerait d'une prise de conscience de la réalité.

\section{Grossesse et consommation}

En accord avec Cassen et al. (2002), la connaissance de la grossesse a entrainé une modification du mode de vie avec notamment la mise en place du traitement de substitution aux opiacés et une rupture avec toutes les relations se rapportant aux consommations chez une majorité de nos sujets.

Ah bah j'vois plus personne toute façon. On voit plus personne. Tout le monde c'était par rapport à ça donc euh. (Natasha)

La grossesse représenterait ainsi la butée qu'elles ne trouvaient pas en leur for intérieur pour se faire aider (Rosenblum, 2009). Même pour Zoé, qui n'était pas dans un désir d'arrêter les consommations, la grossesse et l'enfant ont permis l'investissement dans une prise en charge, la mise en place du traitement et un frein aux consommations malgré plusieurs «rechutes» pendant la grossesse.

La grossesse a également entrainé une diminution importante, voire un arrêt pour certaines, du tabac et/ou du cannabis. L'arrêt ou la limitation des consommations confirme l'idée que la grossesse et le futur bébé induiraient un renflouage narcissique qui rend moins impérieux le besoin de consommer (Nezelof et Dodane, 2010).

Le produit est d'ailleurs, comme l'enfant à naître, source d'ambivalence pour nos sujets excepté pour Cécile et Zoé, pour qui le désir d'arrêter les 
consommations après la grossesse n'est pas présent. Chez ces dernières, la drogue et ses effets délétères sont banalisés, ce qui permet d'en maintenir une vision positive et de mettre à distance toute éventuelle remise en question, et donc tout changement. Les quatre autres sujets oscillent entre des représentations positives et négatives de la drogue. D’un côté, les sujets valorisent — comme la plupart des sujets addicts — le produit du fait des sensations positives et du bien-être qu'il procure. Notamment la suppression de la douleur psychique et du mal-être, des «soucis » et de «tous les problèmes », des pensées négatives, ou encore de l’angoisse.

Et j'me suis dit p'têtre que d'un coup j'oublierai tout et qu'ça irait mieux quoi... Bah c'est l'effet, l'effet qu'ça fait au corps. Ça, le geste et puis... la sensation qu'ça fait quoi. On oublie tout. Et puis on est bien. On est juste... on vit le moment présent. On oublie tout quoi. On a plus aucune notion de, des problèmes qu’on a. (Zoé)

D’un autre côté, nos quatre sujets reconnaissent les conséquences négatives pour l'enfant à naître ainsi que les effets concrets que la dépendance entraine: problèmes financiers, état physique et psychologique, sensation de manque, etc., qui sont une motivation pour arrêter complètement de consommer après la grossesse.

De manière étonnante, le TSO fait l'objet exclusivement de représentations négatives en lien avec les potentielles conséquences sur le bébé (syndrome de sevrage). Karine avait d'ailleurs stoppé complètement son traitement. Elle mettait en avant le bien-être de sa fille pour expliquer son comportement. Soit elle n'a pas compris l'importance de continuer le traitement pendant la grossesse, pourtant expliqué par les médecins, soit elle ne l'a pas entendu en raison de l'angoisse et de la culpabilité massive qu'entraine le fait de savoir que sa fille devra, "par sa faute» être également sous traitement à la naissance. Il se peut également que son bien-être reste au premier plan: "parce que moi j'suis bien [sans le traitement] donc j'ai pas besoin de [drogue]». Il n'est plus question ici du bébé. Plusieurs femmes évoquent également leurs difficultés à gérer TSO et grossesse.

Au-delà des risques effectifs de consommation ou de non-suivi du TSO pendant la grossesse, la question du produit occupe une place très importante dans le discours de ces futures mères. Elle est présente en filigrane dans tous les sujets abordés et signale l'ambivalence entre un désir d'arrêter la consommation et une envie de consommer toujours présente (Lacrouts-Négrier et 
al., 2011). Nous pouvons ainsi avancer l'idée que l'espace psychique maternel de contenance et d'interaction est envahi par la question de la drogue qui reste toujours au centre des préoccupations des femmes usagères de drogues ou sous TSO. Constat qui n'est pas de bon augure car il souligne le risque de troubles dans les relations mère-enfant et ses conséquences sur le développement psychique de l'enfant (Missonnier, 2004).

\section{Mère et fille}

Comme la plupart des femmes, nos sujets vivent leur futur rôle de mère comme source de valorisation sociale (Finkelstein, 1994, cité dans Guyon et al., 1998) mais aussi comme source d'angoisse, s'interrogeant sur leurs capacités maternelles.

Est-ce que je vais être une bonne mère? Est-ce que je vais réussir à bien l'élever? Est-ce que ça en fera quelqu'un de bien? (Véronique)

Leurs consommations et leur dépendance accentuent ces angoisses et ont un impact négatif sur leurs représentations d'elles-mêmes comme sujets et comme mères. Il résulte qu'une partie d'entre elles se voient comme des «mauvaises mères» de par ce qu'elles font «subir» à leur bébé déjà pendant la grossesse.

[Je] trouve difficile le rôle de mère, parce que quand même ça s'engage mal, $[. .$.$] parce que déjà avec tout c'qui va s'passer, là faut... On a beau se$ dire allez, on va tenir le coup, des fois on se dit pauvre petit bébé, il a souffert, il a galéré, la pauvre petite. (Karine)

Pour d'autres, la dévalorisation est masquée par une idéalisation massive qui a pour but de mettre à distance toute représentation négative d'ellesmêmes.

Leurs relations avec leur propre mère influencent également de façon marquée leurs représentations en tant que future mère. Leur enfance et leur adolescence sont marquées par des séparations précoces, des violences physiques et morales, des défaillances parentales importantes ${ }^{7}$ ou bien par des relations paradoxales oscillant entre fusion mère-fille et rejet, abandon.

Moi j'l'ai fait [consommer] parce que je me suis sentie oubliée de mon père. J'me suis sentie aussi oubliée de ma mère. Vu qu'elle me laissait tout 
faire j'me disais euh «J'ai 14 ans. J'suis dans, enfin j'suis pas dans la rue mais... à la limite oui j'étais tous les jours à faire la manche. J'sais pas à 14 ans j'me disais que j'devais pas être là quoi. (Natasha)

Quatre de nos sujets insistent d'ailleurs sur le fait qu'elles ne veulent surtout pas ressembler à leur mère avec leurs propres enfants. Ainsi, dans le discours comme dans l'analyse des planches du Rorschach, l'identification à une «bonne image maternelle» est mise en défaut associée à un désir de différenciation massif (Rosenblum, 2009).

Tout [projection d'elle-même comme mère] sauf comme ma mère! (Zoé)

Or, l'identification à une «bonne image maternelle» suivie d'une différenciation «tempérée » à l'égard de sa propre mère est nécessaire à la femme pour se sentir psychiquement mère (Bayle, 2005). Il y a donc pour ces sujets un risque de ne pouvoir investir la fonction maternelle (Pascal, Spiess et Thevenot, 2011).

Cependant, Karine, Natasha, Charlotte et Zoé tentent de mettre à distance ces ressentis négatifs, peut-être justement dans une tentative de se représenter une image maternelle «suffisamment bonne» afin de pouvoir s'y identifier. De plus, Charlotte semble pouvoir disposer d'autres figures maternelles, support potentiel d'identifications. Véronique, malgré une représentation négative de sa mère, dit souhaiter lui ressembler, soulignant la dimension ambivalentielle et, de ce fait, les potentialités identificatoires et différenciatrices. On repère aussi pour cette dernière une réactivation des conflits infantiles autour de l'autonomie, de la dépendance et de la rivalité par rapport à sa mère. L'élaboration de ces conflits, de bon augure, signalerait une réorganisation du moi féminin en moi maternel (Bibring, 1959). À l'opposé, on observe chez Cécile des représentations uniquement idéalisées chez qui la recherche de différenciation est totalement absente au profit d'un «collage» à la mère.

\section{Registre narcissico-objectal}

Si des défaillances narcissiques apparaissent déjà en filigrane dans le discours de l'ensemble des futures mères interrogées, ce constat est corroboré par l'analyse des planches du Rorschach ${ }^{8}$. Ces défaillances narcissiques se repèrent dans deux registres. D'une part, du fait de l'absence de représentations de relation aux planches dites bilatérales (planches II, III et VII): 
Planche VII :

Ah euh deux personnages $(\mathrm{GF}+\mathrm{H})$. Genre style femmes africaines un peu vous voyez. Ah là je vois 2 chiens weesty aussi oui scottish terrier là (D F- A). (Charlotte)

D'autre part, par l'absence de mouvement pulsionnel ou par des mouvements pulsionnels réprimés:

Planche III :

Deux personnes sur une balançoire. Face à face ( $\mathrm{G} F+\mathrm{H}$ Ban). (Cécile)

On note également une identité acquise mais fragile repérable également sur deux registres. D'une part, la reconnaissance de personnages dans plusieurs planches mais dans une forme régressive avec l'identification d'animaux ou de personnages imaginaires plutôt que d'êtres humains:

Planche II :

Petits éléphants (D F + A Ban). Animaux (D F + A Ban). Trompent qui se touchent (D Kan Ad Ban). Deux éléphants de profil (D F + A Ban). (Karine)

D'autre part par la perception d'êtres humanoïdes, flous:

Planche VII:

Des trucs qui se regardent (D K abst. Tendance choc). C'est pas des bonhommes, c'est des... J'sais pas (Equivalent choc/Refus). Des bonhommes bizarres qui se regardent. Comme des lutins avec un chapeau et une petite queue et qui sont posés sur un truc et qui se regardent (D K (H) Tendance Clob). (Natasha)

On note enfin des troubles de l'identification sexuée et un malaise par rapport à la féminité dans la mesure où la sexuation des personnages humains, lorsqu'ils sont reconnus, est absente:

Planche VII:

Là deux petits enfants qui sont sur un jeu $(\mathrm{G} F+\mathrm{H})$. Qui s'regardent et qui s'parlent (G K H). (Zoé) 
La présence de troubles identitaires implique invariablement des troubles de l'identification.

Le rapport à la féminité est particulièrement en jeu à la planche VII. Des éléments phalliques perçus à cette planche traduisent également un trouble dans l'identité sexuée.

Planche VII:

Alors là non, pas de... (Choc). J'ai l'impression que ça ressemble à des éléphants qui sont sous un chapiteau et qui font leur numéro quoi (G Kan A/scène).» Enquête: «Là on voit sa trompe, son corps qu'est là et le chapiteau. (Véronique)

L'ensemble de ces éléments permet d'affirmer la présence d'une problématique narcissique. Nous devons néanmoins préciser que l'évitement du registre relationnel évoqué ci-dessus pourrait être majoré du fait d'une centration sur soi spécifique au temps de la grossesse.

Le dégagement narcissique vers autrui ne se réalise que sur le mode anaclitique. Les relations à autrui sont déterminées par une dépendance à la mère toujours présente avec l'idée d'un échec du processus de séparationindividuation, problématique prégnante chez les sujets addicts. La mère fait l'objet d'une dévalorisation massive associée à de l'agressivité, mais également d'une demande d'attention, d'intérêt, de réactions.

Quand elle a su pour moi, que j'me droguais euh... [...] ma mère elle a pas réagi euh... en tant que personne normale quoi. Elle s'en fout quoi. Elle dit bah voilà... c'est bien. [...] Elle s'en fout de tout. [silence]. Elle pleure pas. Elle s'en fout. Elle a conscience de rien. [silence]. (Zoé)

La drogue peut d'ailleurs être vue tout autant comme substitut (et maintien) de cette relation de dépendance que comme une tentative, vouée à l'échec, pour en sortir et entrer en relation avec autrui.

La problématique de dépendance anaclitique est également repérable dans la relation au conjoint et père de l'enfant. Pour la plupart de nos sujets, il a une fonction substitutive. Chaque menace sur la relation (disputes ou conflits) conduit d'ailleurs à des reprises de consommation (Guyon et al., 1998). Si Charlotte a été abandonnée dès l'annonce de la grossesse, les cinq autres couples ont tenu bon et quatre des conjoints investissent la fonction d'étayage que ce soit pour le maintien de l'abstinence ou pour la grossesse. 
Un seul, le conjoint de Karine, n'est aucunement étayant. Il est d'ailleurs absent de son discours spontané. Quand il est explicitement questionné dans l'entretien, émergent des représentations soit négatives, soit très idéalisées, peu en lien avec la réalité.

\section{Conclusion}

Notre recherche corrobore l'idée selon laquelle les remaniements psychiques de la grossesse peuvent induire une modification de la dépendance ainsi qu'un investissement libidinal et objectal. Ces transformations dégagent, au moins provisoirement, le sujet de la centration addictive (Cohen-Salmon et al., 2011). Ainsi, la grossesse, par la réactivation des enjeux de passivité et de passivation, ouvrirait la voie à un travail de figuration qui déboucherait sur une remise en question de la relation toxicomane.

Mais, selon Cohen-Salmon et al. (2011), l'importance des remaniements psychiques en jeu ne permettrait à ce processus d'advenir qu'au début ou au cours du troisième trimestre de grossesse, ouvrant tardivement sur un désinvestissement du produit au bénéfice de l'enfant à venir. Propos confirmés par les observations de Lacrouts-Négriet et al. (2001) pour qui le travail psychique de la grossesse ne se ferait que partiellement. Ce ne serait donc qu'en toute fin de grossesse ou au moment de l'accouchement qu'apparaitraient, selon ces auteurs, des réaménagements objectaux potentiels. Plus optimistes, nous pensons que ces processus émergent antérieurement mais qu'ils sont nettement majorés au cours du troisième trimestre. Quoi qu'il en soit, la grossesse serait donc un moment privilégié pour accompagner les femmes usagères de drogues ou sous TSO afin de soutenir ce mouvement.

Au-delà de cette préconisation évidente mais qui demande à être soulignée, notre étude a ouvert de nouvelles interrogations sur l'incidence de ces potentiels réaménagements dans les relations précoces mère-enfant. Nous nous sommes en particulier demandé si les remaniements psychiques observés lors de la grossesse pouvaient être un indicateur prédictif fiable de la qualité des futures relations mère-enfant? Ce nouveau questionnement a donné lieu à une seconde étude, actuellement en cours, sur un suivi longitudinal de femmes toxicomanes à leur septième mois de grossesse et aux trois et douze mois de l'enfant.

Anaëlle Bazire anaelle.bazire@live.fr Nadine Proia-Lelouey 


\section{Notes}

1. Hôpital mère-enfant de l'Est parisien, Association Horizons, ELSA, CH Louis Mourier, CHU Montpellier, réseaux ville-hôpital-toxicomanie, etc.

2. Cette étude a fait l'objet d'une approbation du Comité de protection des personnes (CPP) Bas-Normand. Une présentation orale de l'étude leur a été faite, suivie de la signature d'une lettre de consentement éclairé. La lettre de présentation de la recherche leur avait été envoyée au préalable.

3. Dans le but de garantir l'anonymat des sujets, tous les prénoms ont été modifiés.

4. Et que nous avons retrouvé dans notre clinique en Centre de soins, d'accompagnement et de prévention en addictologie (CSAPA).

5. Ce que nous avons retrouvé dans notre clinique en maternité.

6. L'arrêt du traitement ou des consommations pendant la grossesse entraine un syndrome de sevrage du fotus impliquant les mêmes signes de manque que chez l'adulte et pouvant aboutir à la mort foetale (Drogues info service).

7. Le père de ces femmes est le plus souvent absent ou défaillant.

8. Nous rappelons néanmoins que seule l'analyse du psychogramme et de la totalité des planches permettent de faire des hypothèses sur les problématiques et/ou le fonctionnement psychique de chaque sujet.

\section{Références}

Ammaniti, M., Candelori, C., Pola, M. et Tambelli, R. (1999). Maternité et grossesse: étude des représentations maternelles. Paris: Presses universitaires de France.

Bayle, B. (2005). Enfant à naître, identité conceptionnelle et gestation psychique. Ramonville Saint-Agne: Erès.

Bibring, G.L. (1959). Some considerations of the psychological processes in pregnancy. Psychoanalytic Study of the Child, 14, 113-121.

Bydlowski, M. (1997). La dette de vie, itinéraire psychanalytique de la maternité. Paris: Presses Universitaires de France.

Cassen, M., Facy, F., Pohier, E., Villez, M. et Delile, J.-M. (2002). Maternités et toxicomanies. Étude sur 171 mères toxicomanes et leurs 302 enfants. Bordeaux: Comité d'Étude et d'Information sur la Drogue CEID.

Cohen-Salmon, J., Missonnier, S. et Marty, P. (2011). Addiction et grossesse: du déplacement de l'objet d'addiction vers le nouveau-né. La psychiatrie de l'enfant, 54 (2), 433-468.

Ebert, J. (1988). Maternité-Toxicomanie: Pour un abord plus objectif, le Centre "Horizons". Interventions, 17, 45-50.

Esquivel, T. (1994). Les enfants de parents toxicomanes. Revue Toxicobase, 4, 1-15.

Franchitto, M., Peyrefort, E. et Tellier, G. (2010). Toxicomanie, femmes enceintes et maternité: une nécessaire évolution de la prise en charge. Repéré à: www.hopital-marmottan.fr/spip/ spip.php?article170.

Green, A. (1990). Passions et destins des passions. Dans La folie privée (p.141-193). Paris: Gallimard.

Green, A. (1999). Passivité-passivation: jouissance et détresse. Revue Française de psychanalyse, 63 (5), 1587-1600.

Groupe Pompidou. (2001). Grossesse et toxicomanie, état des lieux en l'an 2000. France: Éditions du Conseil de l'Europe.

Guyon, L., De Konink, M., Morissette, P. et Chayer, L. (1998). Toxicomanie et maternité: un projet possible. Une synthèse des connaissances actuelles: Repéré à http://www.santecom. qc.ca/BibliothequeVirtuelle/CPLT/2550338049.pdf 
Lacrouts-Négrier, N., Roux, C., Taboada, M., Ebert, J. et Schmelck, M-A. (2001). Maternité, interactions mère-enfant et toxicomanie. Centre Horizons et Centre de Psychologie de l'enfance et de l'adolescence de l'Université Paris X-Nanterre.

Lejeune, C., Ropert, J.C. et Montamat, S. (1997). Devenir médico-social de 59 nouveau-nés de mère toxicomane. Journal de Gynécologie, Obstétrique et biologie de la reproduction, 26 (4), 395-404.

Lejeune, C. (2007). Spécificités de prise en charge de certaines populations. Femmes enceintes toxicomanes et périnatalité. Alcoologie et addictologie, 29 (4), 443-448.

McDougall, J. (2004). L'économie psychique de l'addiction. Revue française de psychanalyse, 68 (2), 511-527.

Missonnier, S. (2004). L'enfant du dedans et la relation d'objet virtuel. Dans Missonnier S., Golse B. et Soulé. M. La grossesse, l'enfant virtuel et la parentalité, (p.119-144). Paris: Presses universitaires de France.

Molénat, F. (2000). Grossesse et toxicomanie. Toulouse: Erès.

Nezelof, S. et Dodane, C. (2010). Quand les processus addictifs rencontrent ceux de la parentalité: un climat de turbulences psychiques. Psychotropes, 16 (3-4), 9-16.

Pascal, C., Spiess, M., et Thevenot, A. (2011). Expérience de passivité et affects d'angoisse dans le temps de la grossesse. Dialogue, 192, 137-148.

Proia-Lelouey, N. et Schvan, C. (2011). Processus de maternalité chez les femmes accueillies en centre maternel: de la passivation à la subjectivation. Cahiers de psychologie clinique, 37 (2), 165-179.

Pines D. (1972). Pregnancy and motherhood: interaction between fantasy and reality. British Journal of Medicine Psychology, (45), 333-343.

Richelle, J et al. (2009). Manuel du test du Rorschach. Approche formelle et psychodynamique. Bruxelles: De Boeck.

Rosenblum, O. (2009). Le féminin maternel chez les femmes toxicomanes sous substitution. De la passion du corps à la resexualisation de la relation. Dialogue, 184 (2), 115-129.

Simmat-Durand, L. (2002). Grossesse et drogues illicites. Déviance et société, 26 (1), 105-126.

Soulé, M. (1982). L'enfant imaginaire: fantasme d'enfant, le désir d'enfant, le désir de grossesse. Dans T.B. Brazelton et al. (dir.), La dynamique du nourrisson (p. 137-175). Paris: ESF.

Taboada, M-J. (1988). Aspects psychopathologiques des femmes usagères de drogues. Berne: Symposium du Groupe Pompidou.

Toubiana, E.-P. (2011). Addictologie clinique. Paris: Presses universitaires de France.

Touzanne, B. (2009). Accroche-moi et «accroche-toi». Addictions à l'adolescence, une histoire du lien. La lettre de l'enfance et de l'adolescence, (77), 71-76. 


\section{Annexe: Présentation globale des sujets}

Les six femmes incluses dans cette recherche étaient âgées de 22 à 33 ans. Quatre d'entre elles attendaient un enfant de sexe féminin. L'une d'entre elles était par ailleurs déjà mère d'une première fille âgée de deux ans et demi.

Quatre des six conjoints étaient consommateurs d'héroïne et/ou sous TSO. Deux de ces quatre hommes étaient présents et représentaient un soutien important pour les futures mères pendant la grossesse. Le troisième conjoint vivait avec sa compagne mais était absent de son discours et ne semblait pas investi dans la grossesse, ni représenter un soutien pour la future mère. Enfin, le quatrième était absent, ayant abandonné sa compagne à l'annonce de la grossesse. Deux des six futurs pères étaient donc non-consommateurs. Ces derniers étaient présents et soutenants pendant la grossesse mais l'un d'eux était également très culpabilisant avec sa compagne par rapport aux consommations (drogues et TSO) pendant la grossesse.

L'on notait par ailleurs une dépendance affective majeure de trois des six femmes à l'égard de leur conjoint.

Toutes les femmes consommaient de l'hérö̈ne avant la connaissance de la grossesse, associée à des consommations de tabac (toutes) et/ou de cannabis (quatre femmes). Deux femmes avaient également recours à la cocaïne.

Trois des femmes avaient déjà entamé un suivi et mis en place un traitement de substitution aux opiacés (TSO) avant la connaissance de la grossesse. Celui-ci a été poursuivi pendant la grossesse. Les trois autres femmes ont mis en route le TSO à la connaissance de leur grossesse, associé à un arrêt complet des consommations d'héroïne pour deux de ces trois femmes. Plusieurs des six femmes ont également réduit ou arrêté les consommations de cannabis et de tabac. Deux femmes consommaient occasionnellement de l'héroïne, en plus du TSO, pendant leur grossesse.

L'âge du début des consommations d'héroïne apparait disparate entre les femmes (13 ans à 28 ans). Néanmoins, l'on repère, chez quatre d'entre elles, un début de consommations de drogues (cannabis) dès le début de l'adolescence (13-14 ans). 